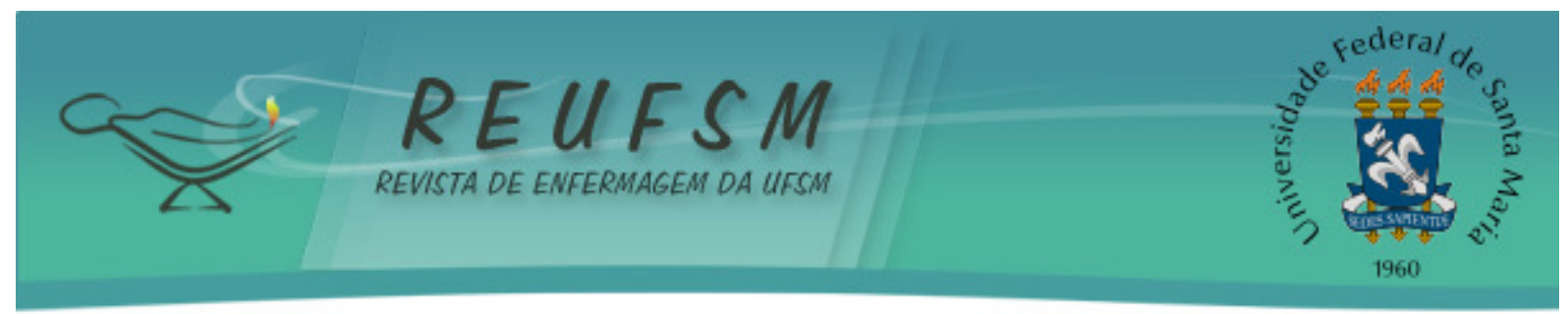

ARTIGO ORIGINAL

\title{
CARACTERIZAÇÃO DE PACIENTES EM TRATAMENTO RADIOTERÁPICO A PARTIR DA CONSULTA DE ENFERMAGEM
}

CHARACTERIZATION OF PATIENTS IN RADIOTHERAPICAL TREATMENT FROM THE
NURSING CONSULTATION

\section{CARACTERIZACIÓN DE LOS PACIENTES EN TRATAMIENTO RADIOTERAPÉUTICO A} PARTIR DE LA CONSULTA DE ENFERMERÍA

\author{
Camila Marcon ${ }^{1}$ \\ Carla Argenta ${ }^{2}$ \\ Fernanda Karla Metelski ${ }^{3}$ \\ Vanessa Schorr ${ }^{4}$ \\ Édlamar Kátia Adamy ${ }^{5}$
}

Doi: $10.5902 / 2179769224914$

RESUMO: Objetivo: caracterizar o perfil sociodemográfico e clínico de pacientes em tratamento radioterápico, a partir da consulta de enfermagem. Método: estudo quantitativo, do tipo exploratório descritivo realizado em um ambulatório de radioterapia, com 60 pacientes em tratamento radioterápico, com idade igual ou superior a 18 anos. A coleta de dados ocorreu nos meses de junho e julho de 2015 e foram analisados por estatística descrita. Resultados: a maioria dos pacientes foi do sexo feminino, com idade entre 50 e 79 anos, com ensino fundamental completo, casados e com 1 a 3 filhos, sendo o câncer de mama em mulheres e o de próstata em homens, os mais incidentes. Considerações finais: a consulta de enfermagem mostrou-se uma estratégia de trabalho eficiente. $O$ estudo pode contribuir para a elaboração de protocolos assistenciais que possibilitam qualificar o cuidado prestado.

Descritores: Enfermagem; Oncologia; Radioterapia.

ABSTRACT: Aim: to characterize the sociodemographic and clinical profile of patients in radiotherapical treatment from the nursing consultation. Method: this is a quantitative, descriptive exploratory study carried out in a radiotherapy outpatient clinic, with 60 patients undergoing radiotherapy treatment aged 18 years or older. The data analysis was made through descriptive statistics and its collection occurred during June and July 2015. Results: the majority of the patients were female, aged between 50 and 79 years old, with complete primary education, married and with 1 to 3 children. The most incidental outcomes were breast cancer in women and prostate cancer in men. Final considerations: nursing consultation proved to be an efficient work strategy. The study can contribute to the elaboration of assistance protocols that allows qualifying the care provided.

Descriptors: Nursing; Medical oncology; Radiotherapy.

\footnotetext{
${ }^{1}$ Enfermeira. Universidade do Estado de Santa Catarina (UDESC), Chapecó, Santa Catarina (SC), Brasil. e-mail: milamarcon_md@hotmail.com

2 Enfermeira, Professora Assistente da UDESC, Doutoranda em Enfermagem pela Universidade Federal do Rio Grande do Sul (UFRGS), Chapecó, Santa Catarina (SC), Brasil. e-mail: carlaargenta@yahoo.com.br

${ }^{3}$ Enfermeira, Professora Assistente da UDESC, Mestre em Políticas Sociais e Dinâmicas Regionais, Chapecó, Santa Catarina (SC), Brasil. e-mail: fernanda.metelski@gmail.com

${ }^{4}$ Enfermeira, Universidade do Estado de Santa Catarina (UDESC), Chapecó, Santa Catarina (SC), Brasil. e-mail: vanessa.schorr@yahoo.com

${ }^{5}$ Enfermeira, Professora Adjunta da UDESC, Doutora em Enfermagem UFRGS, Chapecó, Santa Catarina (SC), Brasil. e-mail: edlamar.adamy@udesc.br
} 


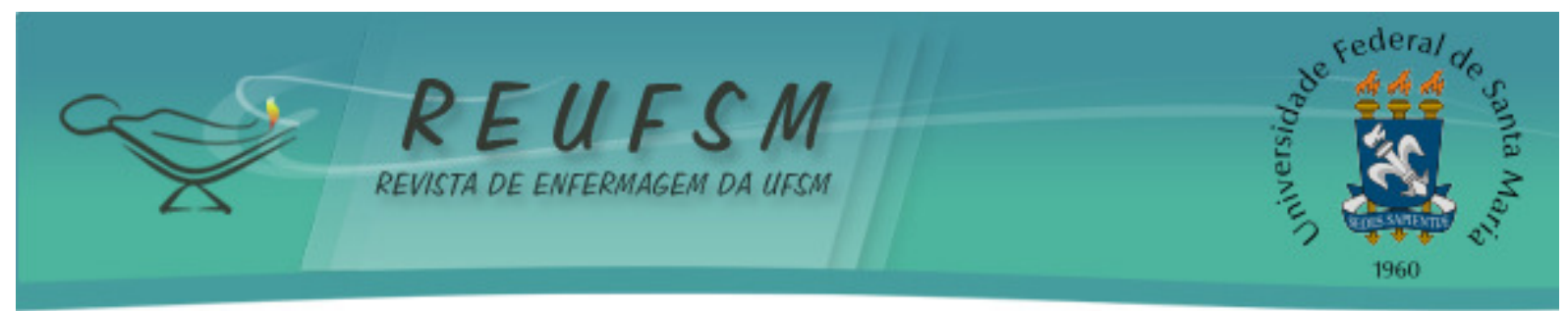

RESUMEN: Objetivo: caracterizar el perfil sociodemográfico y clínico de los pacientes en tratamiento radioterapéutico a partir de la consulta de enfermería. Método: estudio cuantitativo, exploratorio, descriptivo realizado en un ambulatorio de radioterapia, con 60 pacientes en tratamiento radioterapéutico, con edades iguales o mayores que 18 años. La recolección de datos ocorrió durante los meses de junio y julio de 2015 y fueron analizados por estadística descrita. Resultados: la mayoría de los pacientes eran mujeres, con edades entre 50 y 79 años, con la escuela primaria completa, casados y con 1 a 3 hijos, el mayor número de incidencia es el cáncer de mama entre las mujeres y próstata entre los hombres. Consideraciones finales: la consulta de enfermería demostró ser una estrategia de trabajo eficiente. El estudio puede contribuir para el desarrollo de protocolos asistenciales, que pueden calificar el cuidado ofrecido.

Descriptores: Enfermería; Oncología médica; Radioterapia.

\section{INTRODUÇÃOO}

O câncer é considerado uma doença crônica e estima-se para o ano de 2020, 553.050 novos casos de câncer para o Brasil, 3.558.826 para a região da Américas e 17.113.588 em todo o mundo e que, destes, aproximadamente $50 \%$ precisarão de tratamento radioterápico. ${ }^{1-3}$

0 avanço das terapias individualizadas tem prolongado a vida dos pacientes, sendo que o tratamento para o câncer pode ser realizado de forma local (cirurgia e radioterapia), ou sistêmica (quimioterapia), havendo para cada tipo de câncer e seu agravo, uma indicação de tratamento. A radioterapia é caracterizada por ser uma das grandes modalidades de tratamento para as neoplasias, por usar radiação ionizante que é produzida por aparelhos ou que pode ser emitida por radioisótopos naturais realizada, na maioria das vezes, em regime ambulatorial. ${ }^{1}$ É indicada em mais de $50 \%$ dos casos nos países em desenvolvimento. Dentre os pacientes curados, aproximadamente $49 \%$ são por cirurgias, $40 \%$ por tratamento com radioterapia isolada ou combinada com outras modalidades de tratamento, e $11 \%$ por quimioterapia isolada ou combinada. ${ }^{4}$

Considerando este cenário, o enfermeiro tem por função atuar de maneira efetiva durante o tratamento radioterápico, na redução dos efeitos adversos, prestando cuidados específicos e adequados para cada necessidade dos pacientes. Diante disso, a consulta de enfermagem possibilita obter informações que subsidiem a tomada de decisão, para qualificar a assistência, com vistas ao autocuidado e suporte para enfrentar o tratamento. ${ }^{1}$

No ambulatório de radioterapia, a consulta de enfermagem tem se demonstrado eficaz na execução de intervenções e na prevenção de complicações provenientes do tratamento. Destaca-se que, no hospital onde foi desenvolvido o estudo, foram realizados 425.890 procedimentos de radioterapia entre os anos de 2011 e 2016.5 No município de Chapecó, Santa Catarina, as neoplasias atingiram $28,49 \%$ do total de óbitos entre os anos de 2012 e 2016 e, portanto, constituem a primeira causa de óbito, com uma prevalência de $82,88 \%$ para a faixa etária de 50 anos ou mais. ${ }^{6}$

Neste contexto, o número de pacientes que vem sendo atendidos e o quantitativo de óbitos por esta causa evidencia a abrangência do serviço e desperta o interesse em conhecer esse público, a fim de qualificar as intervenções de enfermagem. Desta forma, propôs-se a seguinte questão norteadora: qual o perfil sociodemográfico e clínico de pacientes em tratamento radioterápico?

Portanto, o presente estudo objetiva caracterizar o perfil sociodemográfico e clínico de pacientes em tratamento radioterápico, a partir da consulta de enfermagem. 


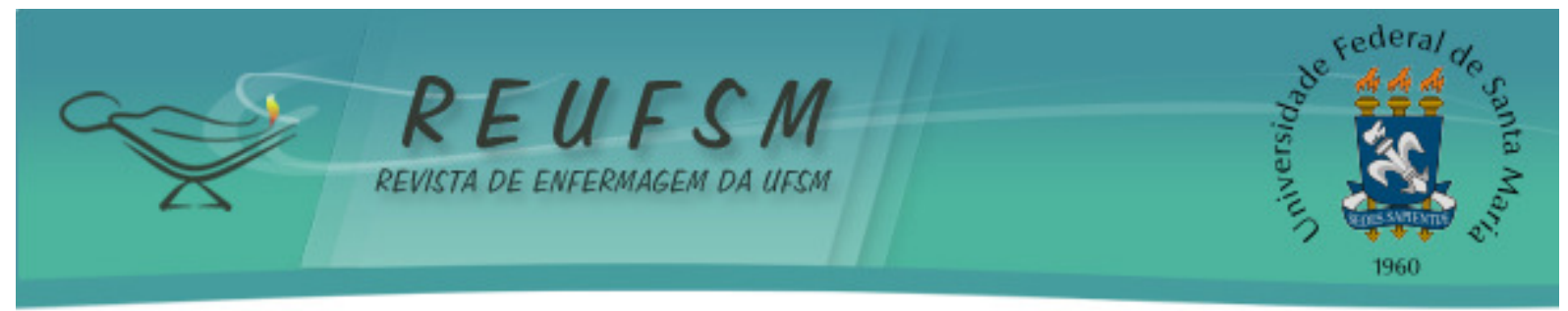

MÉTODO

Trata-se de um estudo quantitativo, do tipo exploratório descritivo realizado no ambulatório de radioterapia de um hospital de referência que atende as regiões Oeste e Meio Oeste do Estado de Santa Catarina, localizado na cidade de Chapecó. Foram incluídos 60 pacientes em tratamento radioterápico com idade igual ou superior a 18 anos, de ambos os sexos, independente do município de origem. Foi excluído um paciente em condição cognitiva prejudicada, avaliado por meio do teste de fluência verbal que fornece informações acerca da capacidade de armazenamento do sistema de memória semântica e aparece alterada em múltiplos processos patológicos, tais como as demências degenerativas. ${ }^{7}$

Os pacientes que cumpriram os critérios de inclusão foram convidados a participar da pesquisa mediante assinatura do Termo de Consentimento Livre e Esclarecido.

Para a coleta de dados utilizou-se de um instrumento construído para este estudo e a mesma ocorreu durante a consulta de enfermagem, no ambulatório de radioterapia, realizada pela enfermeira responsável pelo setor, nos meses de junho e julho de 2015, com tempo médio de uma hora para cada consulta.

0 instrumento de coleta de dados foi elaborado com questões sociodemográficas que incluem: sexo, faixa etária, escolaridade, estado civil e número de filhos; e questões clínicas relacionadas ao tipo de câncer com destaque para o fator de risco tabagismo. Para realizar a compilação e análise estatística descritiva, utilizou-se o programa Microsoft Excel $^{\circledR} 2010$ seguido do programa Epilnfo ${ }^{T M} 7$.

Este estudo foi aprovado pelo Comitê de Ética em Pesquisa com Seres Humanos da Universidade do Estado de Santa Catarina, sob o número 1.087.561 de 29/05/2015, e conduzido de acordo com os padrões éticos estabelecidos pela Resolução 466/12 do Conselho Nacional de Saúde.

\section{RESULTADOS}

Participaram desse estudo 60 pacientes, predominantemente mulheres $(56,7 \%)$, na faixa etária entre 50 e 79 anos de idade $(71,7 \%)$, com ensino fundamental completo $(45 \%)$ e casados(as) (65\%) (Tabela 1).

Tabela 1 - Distribuição das variáveis sociodemográficas dos pacientes submetidos ao tratamento radioterápico. Chapecó, 2015. ( $\mathrm{N}=60)$

\begin{tabular}{lcc}
\hline \multicolumn{1}{c}{ Variáveis } & N & $\%$ \\
\hline Sexo & 34 & 56,7 \\
Feminino & 26 & 43,3 \\
Masculino & 10 & 16,6 \\
Faixa etária & 43 & 71,7 \\
20 a 49 anos & 7 & 11,7 \\
50 a 79 anos & & \\
80 a 99 anos & 6 & 10 \\
Escolaridade & 13 & 21,6 \\
Analfabetos & 27 & 45 \\
EFI $^{1}$ & 3 & 5 \\
EFC $^{2}$ & 7 & 11,7 \\
EMI $^{3}$ & 1 & 1,7 \\
EMC $^{4}$ & 2 & 3,3 \\
ESI $^{5}$ & 1 & 1,7 \\
ESC $^{6}$ & &
\end{tabular}




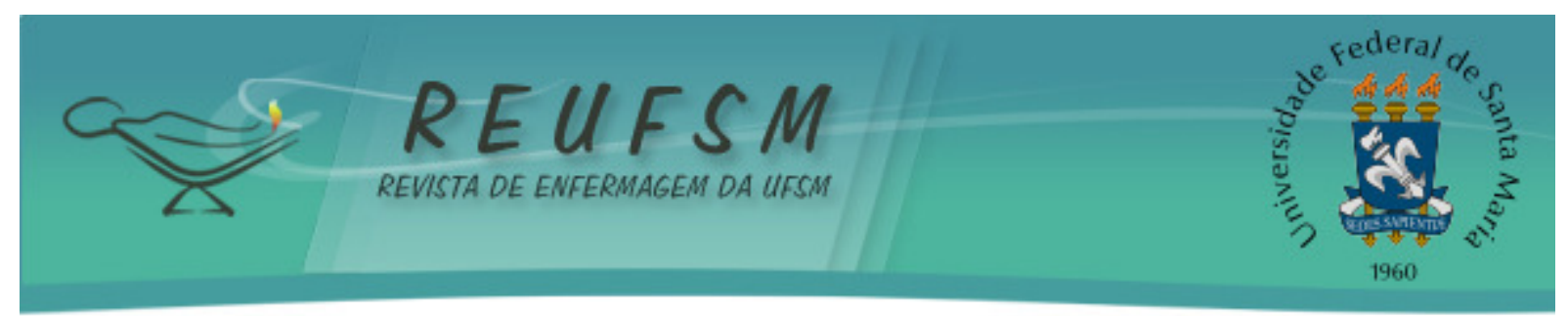

\begin{tabular}{lcc} 
Estado Civil & & \\
Solteiros & 2 & 3 \\
Casados & 39 & 65 \\
União Estável & 3 & 5 \\
Divorciados & 8 & 13 \\
Viúvos & 8 & 14 \\
\hline Número de Filhos & & \\
1 a 3 & 28 & 46,7 \\
4 a 6 & 19 & 31,7 \\
7 ou mais & 9 & 15 \\
Nenhum & 4 & 6,6 \\
\hline
\end{tabular}

${ }^{1}$ Ensino fundamental incompleto; ${ }^{2}$ Ensino fundamental completo; ${ }^{3}$ Ensino médio incompleto; ${ }^{4}$ Ensino médio completo; ${ }^{5}$ Ensino superior incompleto; ${ }^{6}$ Ensino superior completo.

Para o sexo feminino, destaca-se que o tipo de câncer encontrado nos pacientes em tratamento radioterápico centra-se no de mama, acometendo 19 pacientes (56\%), na faixa etária entre 30 e 79 anos de idade, seguido do câncer do colo uterino, encontrado em seis pacientes (17\%) na faixa etária que variou de 40 até 79 anos de idade.

$\mathrm{Na}$ tabela 2 estão representados os tipos de câncer que acometeram os pacientes submetidos ao tratamento radioterápico, atendidos durante a consulta de enfermagem.

Tabela 2 - Distribuição dos tipos de câncer segundo o sexo para os pacientes submetidos a tratamento radioterápico, Chapecó, $2015(\mathrm{~N}=60)$.

\begin{tabular}{|c|c|c|c|c|c|c|c|}
\hline & Variáveis & Masc & ino & Fem & lino & & tal \\
\hline $\begin{array}{l}\text { Tipo } \\
\text { Câncer }\end{array}$ & & $\mathrm{N}$ & $\%$ & $\mathrm{~N}$ & $\%$ & $\mathrm{~N}$ & $\%$ \\
\hline & $\mathrm{CA}^{1}$ próstata & 9 & 34 & 0 & 0 & 9 & 15 \\
\hline & $\mathrm{CA}^{1}$ reto & 4 & 15 & 0 & 0 & 4 & 6,6 \\
\hline & $\mathrm{CA}^{1}$ esôfago & 1 & 4 & 0 & 0 & 1 & 1,7 \\
\hline & $C A^{1}$ laringe & 2 & 7 & 0 & 0 & 2 & 3,3 \\
\hline & $\mathrm{CA}^{1}$ pele & 2 & 8 & 2 & 6 & 4 & 6,6 \\
\hline & $\mathrm{CA}^{1}$ orofaringe & 1 & 4 & 0 & 0 & 1 & 1,7 \\
\hline & $\mathrm{CA}^{1}$ base de língua & 1 & 4 & 0 & 0 & 1 & 1,7 \\
\hline & $\mathrm{CA}^{1}$ pulmão & 2 & 8 & 0 & 0 & 2 & 3.3 \\
\hline & CA ${ }^{1}$ língua & 1 & 4 & 0 & 0 & 1 & 1,7 \\
\hline & $\mathrm{CA}^{1}$ cervical & 1 & 4 & 0 & 0 & 1 & 1,7 \\
\hline & CA EMDURC $^{2}$ & 2 & 8 & 0 & 0 & 2 & 3,3 \\
\hline & $\mathrm{CA}^{1}$ mama & 0 & 0 & 19 & 56 & 19 & 31,6 \\
\hline & $\mathrm{CA}^{1}$ colo uterino & 0 & 0 & 6 & 17 & 6 & 10 \\
\hline & CA pulmão & 0 & 0 & 3 & 9 & 3 & 5 \\
\hline & $\mathrm{CA}^{1}$ linfoma & 0 & 0 & 1 & 3 & 1 & 1,7 \\
\hline & $C A^{1}$ bexiga & 0 & 0 & 1 & 3 & 1 & 1,7 \\
\hline & $\mathrm{CA}^{1}$ pâncreas & 0 & 0 & 1 & 3 & 1 & 1,7 \\
\hline & $\mathrm{CA}^{1}$ cabeça/pescoço & 0 & 0 & 1 & 3 & 1 & 1,7 \\
\hline & Total & 26 & 100 & 34 & 100 & 60 & 100 \\
\hline
\end{tabular}

${ }^{1}$ Câncer; ${ }^{2}$ Câncer em mais de uma região do corpo 


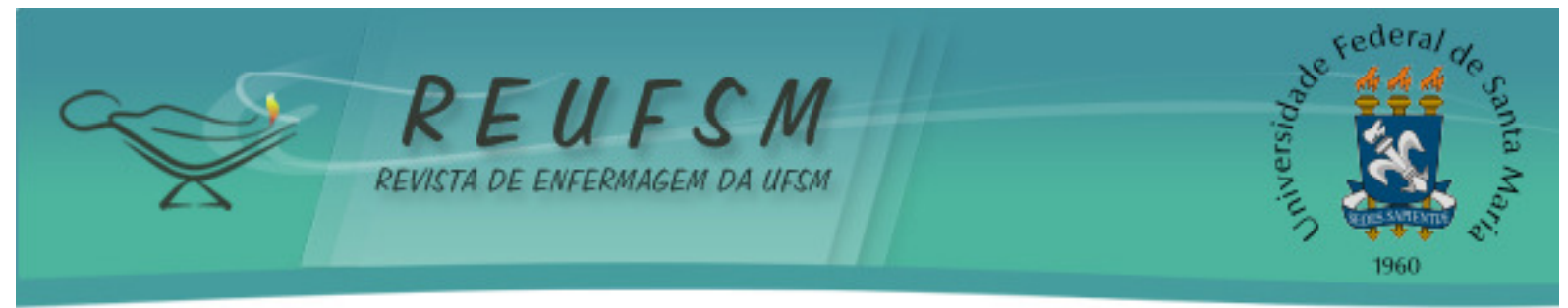

Pela expressiva relação do tabagismo e os casos de câncer, apresenta-se a tabela 3 que descreve esta distribuição.

Tabela 3 - Distribuição dos tipos de cânceres segundo o tabagismo e sexo entre os pacientes atendidos, Chapecó, 2015 ( $\mathrm{N}=60)$.

\begin{tabular}{|c|c|c|c|c|}
\hline \multirow{2}{*}{$\begin{array}{l}\text { Variáveis } \\
\text { Tipo de câncer }\end{array}$} & \multicolumn{2}{|c|}{ Tabagista - Masculino } & \multicolumn{2}{|c|}{ Tabagista - Feminino } \\
\hline & SIM & NÂO & SIM & NÃO \\
\hline CA'Próstata & 7 & 2 & 0 & 0 \\
\hline$C A^{1}$ Reto & 3 & 1 & 0 & 0 \\
\hline CA ${ }^{1}$ Esôfago & 1 & 0 & 0 & 0 \\
\hline$C A^{1}$ Laringe & 2 & 0 & 0 & 0 \\
\hline $\mathrm{CA}^{1}$ Pele & 1 & 1 & 0 & 2 \\
\hline $\mathrm{CA}^{1}$ Orofaringe & 1 & 0 & 0 & 0 \\
\hline $\mathrm{CA}^{1}$ Base de língua & 0 & 1 & 0 & 0 \\
\hline $\mathrm{CA}^{1}$ Pulmão & 2 & 0 & 2 & 1 \\
\hline CA ${ }^{1}$ Língua & 0 & 1 & 0 & 0 \\
\hline $\mathrm{CA}^{1}$ Cervical & 1 & 0 & 0 & 0 \\
\hline $\begin{array}{l}\mathrm{CA}^{1} \text { em mais de uma parte do } \\
\text { corpo }\end{array}$ & 2 & 0 & 0 & 0 \\
\hline $\mathrm{CA}^{1}$ Mama & 0 & 0 & 5 & 14 \\
\hline $\mathrm{CA}^{1}$ Colo uterino & 0 & 0 & 1 & 5 \\
\hline$C A^{1}$ Bexiga & 0 & 0 & 0 & 1 \\
\hline$C A^{1}$ Linfoma & 0 & 0 & 0 & 1 \\
\hline CA $A^{1}$ Pâncreas & 0 & 0 & 1 & 0 \\
\hline $\mathrm{CA}^{1}$ Cabeça/pescoço & 0 & 0 & 1 & 0 \\
\hline Total & 20 & 6 & 10 & 24 \\
\hline
\end{tabular}

\section{DISCUSSÃO}

Para muitos tipos de câncer, a idade avançada é caracterizada como um fator de risco significativo. Há uma grande influência da idade para alterações que ocorrem no corpo humano, a exemplo das alterações hormonais. ${ }^{8}$

Em estudo realizado com idosos chineses mostrou a predominância masculina de cânceres em geral e os prognósticos ruins têm sido atribuídos ao atraso nos diagnósticos. ${ }^{9}$

Os dados desta pesquisa apontam que o tipo de câncer que mais acomete os homens em tratamento radioterápico é o câncer de próstata. Dados do Instituto Nacional do Câncer (INCA) reforçam os resultados dessa pesquisa, pois apontam que o segundo tipo de câncer mais frequente em homens foi o de próstata, com cerca de 61.200 casos novos no ano de 2016. ${ }^{10}$

Os casos de câncer de próstata apresentaram-se entre os principais tipos de câncer que acometeram a população masculina para a faixa etária de 60 a 89 anos. Este achado corrobora com estudo que aponta a idade como fator de risco para o desenvolvimento do câncer de próstata, pois cerca de $62 \%$ dos casos que são diagnosticados no mundo, ocorrem em homens na faixa etária de 65 anos ou mais. ${ }^{8}$

O câncer de próstata é considerado o mais comum entre os homens na Europa e na América do Norte, no entanto a identificação robusta de fatores de risco potencialmente modificáveis tem se mostrado relevantes para a realização de estudos. ${ }^{11}$

Nesta pesquisa, o câncer de reto, foi evidenciado em pacientes com idade entre 50 e 69 anos, dado este que corrobora com estudos publicados apontando o câncer de cólon e reto como o terceiro tipo mais comum encontrado no sexo masculino. 0 câncer de cólon e 


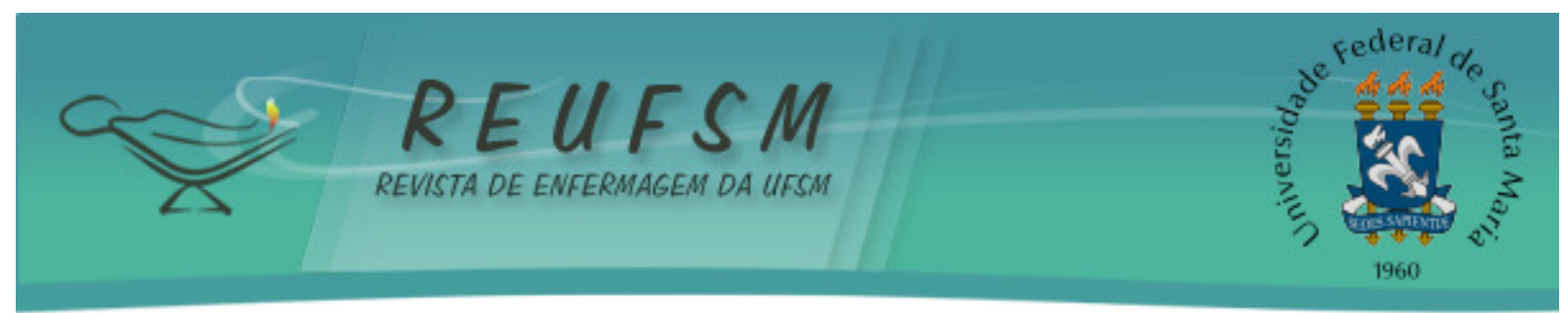

reto tem estimativa, para o Brasil, de 16.660 novos casos em homens e 17.620 em mulheres. Este tipo de neoplasia é a segunda mais frequente na Região Sudeste $(24,27 / 100$ mil), terceira na Região Sul (22,35/100 mil) e quarta no Centro-Oeste (14,16/100 mil). ${ }^{3,8,12}$

Para o biênio 2016-2017 no Brasil, aponta-se a ocorrência de cerca de 600 mil casos novos de câncer. Excluindo a neoplasia de pele não melanoma (aproximadamente 180 mil casos novos), estão previstos cerca de 420 mil casos novos de câncer. Desconsiderando os casos de câncer de pele não melanoma, os tipos mais frequentes em homens serão próstata $(28,6 \%)$, pulmão $(8,1 \%)$, intestino $(7,8 \%)$, estômago $(6,0 \%)$ e cavidade oral $(5,2 \%)$. Já nas mulheres, serão os cânceres de mama $(28,1 \%)$, intestino $(8,6 \%)$, colo do útero $(7,9 \%)$, pulmão $(5,3 \%)$ e estômago $(3,7 \%) .^{12}$

O tabaco é um grande fator de risco para o desenvolvimento de vários tipos de neoplasias, principalmente para os cânceres de pulmão, cavidade oral, laringe, faringe, esôfago, estômago, pâncreas, fígado, rim, bexiga, colo do útero e leucemias. As pessoas que fumam possuem um risco maior de desenvolver este tipo de doença, quando comparadas aos não fumantes. ${ }^{8}$

O câncer que mais acomete o sexo feminino, em todo o mundo, é o de mama, também responsável pelo maior número de mortes por esta doença em mulheres. Vários são os fatores de risco que predispõe para o desenvolvimento desta doença, contudo, o principal fator é a idade, evidenciado em mulheres com idade acima de 50 anos. ${ }^{8}$

No Brasil, para o ano de 2014, foi estimado cerca de 15.590 casos novos de câncer de colo de útero. Este tipo de câncer é configurado como um importante problema de saúde pública, considerado o quarto tipo de neoplasia mais comum encontrado no sexo feminino. Esta doença acomete mulheres a partir dos 30 anos de idade, e seu risco aumenta de forma rápida até atingir o pico da faixa etária entre 50 a 60 anos. ${ }^{8}$

Para as mulheres, a variável número de filhos está associada ao câncer de mama feminino, uma vez que esse tipo de neoplasia é considerado fator de risco para mulheres que engravidam pela primeira vez após os 30 anos de idade ou aquelas que são nulíparas. ${ }^{13}$ Segundo estudo, os principais fatores para o desenvolvimento do câncer de mama estão relacionados ao sistema reprodutivo, principalmente a menarca precoce, menopausa tardia, a nuliparidade, oligoparidade, assim como a primeira gestação tardia. ${ }^{14}$

Conforme estudo com dados de 47 investigações epidemiológicas em 30 países, o risco de desenvolver câncer de mama diminui com o aumento da duração da amamentação, sendo esta redução de $4,5 \%$ para cada ano de amamentação. Já nos casos das mulheres nulíparas, considerando que estas não se beneficiarem do efeito protetor que é a amamentação, orienta-se e estimula-se para que adotem hábitos saudáveis visando a prevenção de fatores de risco que estão associados ao desenvolvimento desta neoplasia. ${ }^{15}$

\section{CONSIDERAÇÕES FINAIS}

Para a caracterização sociodemográfica e clínica dos pacientes em tratamento radioterápico, a consulta de enfermagem mostrou-se uma estratégia de trabalho eficiente que possibilita conhecer os pacientes para a tomada de decisão acurada em relação às intervenções pertinentes.

Os resultados referentes à caracterização sociodemográfica mostram que a maioria dos pacientes é do sexo feminino, com idade entre 50 e 79 anos, com ensino fundamental completo, casados e com 1 a 3 filhos.

Os resultados referentes à caracterização clínica apontam que o câncer de mama em mulheres e o de próstata em homens são os mais incidentes. Merece destaque, a relação da presença de câncer e o avançar da idade. 


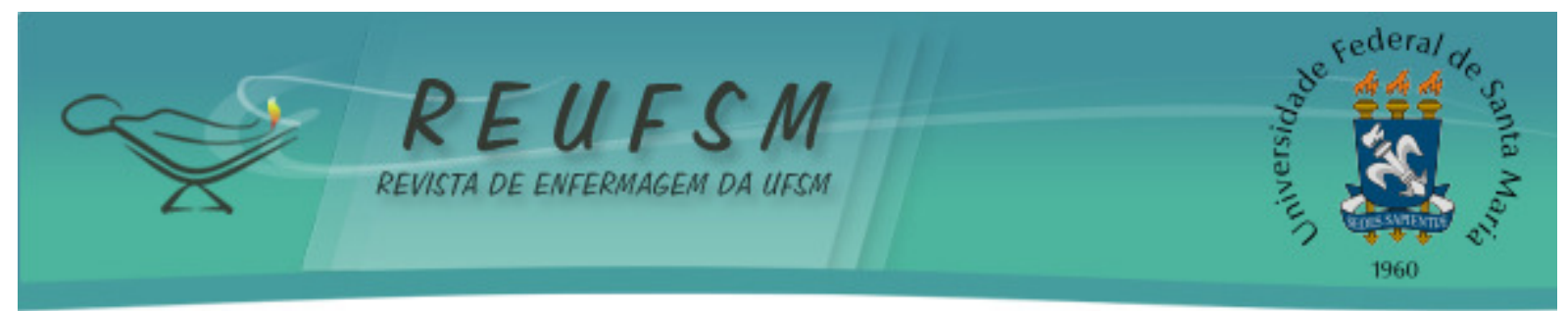

O presente estudo pode contribuir para a elaboração de protocolos assistenciais que possibilitem qualificar o cuidado prestado, considerando a necessidade de ampliação, para além do serviço de radioterapia, avançando para as Redes de Atenção à Saúde, com foco na regionalização.

\section{REFERÊNCIAS}

1. Leite FMC, Ferreira FM, Cruz MSA, Lima EFA, Primo CC. Diagnósticos de enfermagem relacionados aos efeitos adversos da radioterapia. REME Rev Min Enferm [Internet]. 2013 [acesso em 2016 out 8];17(4):940-5. Disponível em: http://www.reme.org.br/artigo/detalhes/897.

2. Facina T. Estimativa 2014: incidência de câncer no Brasil. Rev Bras Cancerol [Internet]. 2014 [acesso em 2016 set 11] 60(1):63-4. Disponível em: http: / / www1.inca.gov.br/rbc/n_60/v01/pdf/11-resenha-estimativa-2014-incidencia-decancer-no-brasil.pdf.

3. Organização Mundial da Saúde (OMS). Globocan 2012: estimated cancer incidence, mortality and prevalence woldwide in 2012 [Internet]. 2012 [acesso em 2017 abr 17]. Disponível em: http://globocan.iarc.fr/Pages/burden_sel.aspx.

4. International Atomic Energy Agency (IAEA). Planning national radiotherapy services: practical tool [Internet]. Vienna. 2010 [acesso em 2017 abr 17]. Disponível em: http: / /www-pub.iaea.org/books/IAEABooks/8419/Planning-National-Radiotherapy-

Services-A-Practical-Tool. (IAEA Human Health Series; 14).

5. Brasil. Ministério da Saúde. Datasus. Produção Ambulatorial do SUS - Santa Catarina Por Local de Atendimento [Internet]. 2017 [acesso em 2017 abr 16]. Disponível em: http://tabnet.datasus.gov.br/cgi/tabcgi.exe?sia/cnv/qasc.def.

6. Brasil. Ministério da Saúde. Datasus. Sistema de Informações sobre Mortalidade - SIM. [Internet]. 2017 [acesso em 2017 abr 16]. Disponível em: http://www.saude.sc.gov.br/cgi/deftohtm.exe?sim96.def.

7. Brucki SMD, Malheiros SMF, Okamoto IH, Bertolucci PHF. Dados normativos para o teste de fluência verbal categoria animais em nosso meio. Arq Neuro-psquiatr. 1997;55(1):56-61.

8. Brasil. Ministério da Saúde. Instituto Nacional de Câncer José Alencar Gomes da Silva (INCA). Estimativa 2014: incidência de câncer no Brasil. Rio de Janeiro (RJ): INCA. [internet]. 2014 [acesso em 2016 set 11]. Disponível em: http://www.inca.gov.br/bvscontrolecancer/publicacoes/Estimativa_2014.pdf.

9. Higuera O, Ghanem I, Nasimi R, Prieto I, Koren L, Feliu J. Management of pancreatic cancer in the elderly. World J Gastroenterol [Internet]. 2016 [acesso em $2017 \mathrm{abr}$ 16];22(2):764-75. Disponível https://www.ncbi.nlm.nih.gov/pmc/articles/PMC4716075/.

10. Brasil. Ministério da Saúde. Instituto Nacional de Câncer José Alencar Gomes da Silva (INCA). Próstata [internet]. 2017 [acesso em 2017 abr 16]. Disponível em: http://www2.inca.gov.br/wps/wcm/connect/tiposdecancer/site/home/prostata/definicao.

11. Davies NM, Gaunt TR, Lewis SJ, Holly J, Donovan JL, Hamdy FC, et al. The effects of height and $\mathrm{BMI}$ on prostate cancer incidence and mortality: a Mendelian randomization study in 20,848 cases and 20,214 controls from the PRACTICAL consortium. Cancer Causes 


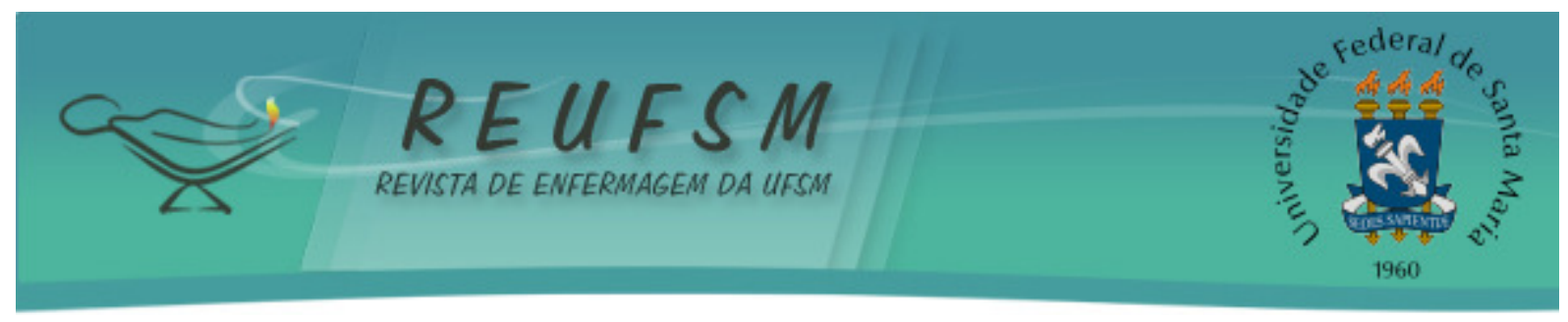

Control [Internet]. 2015 [acesso em 2017 abr 16];26(11):1603-16. Disponível em: https://www.ncbi.nlm.nih.gov/pmc/articles/PMC4596899/.

12. Brasil. Ministério da Saúde. Instituto Nacional de Câncer José Alencar Gomes da Silva (INCA). Estimativa 2016: incidência de câncer no Brasil [Internet]. Rio de Janeiro (RJ): INCA; 2015 [acesso em 2016 set 26]. Disponível em: http://www.inca.gov.br/estimativa/2016/estimativa-2016-v11.pdf.

13. Fundação do Câncer; Instituto Nacional do Câncer José Alencar Gomes da Silva (INCA). Colorretal [Internet]. Rio de Janeiro (RJ): Fundação do Cancer; INCA: 2015 [acesso em 2016 out 11]. Disponível em: //cancer.org.br/sobre-o-cancer/tipos-de-cancer/colum-e-reto/.

14. Nazário ACP, Facina G, Filassi JR. Breast cancer: news in diagnosis and treatment. Rev Assoc Med Bras [Internet]. 2015 [acesso em 2017 abr 15];61(6):543-52. Disponível em: http://www.scielo.br/scielo.php?script=sci_arttext\&pid=S0104-42302015000600543.

15. Collaborative Group on Hormonal Factors in Breast Cancer. Breast cancer and breast feeding: collaborative reanalysis of individual data from 47 epidemiological studies in 30 countries, including 50302 women with breast cancer and 96973 women without the disease. Lancet. 2002;360(9328):187-95.

Data de recebimento: $27 / 11 / 2016$

Data de aceite: 31/05/2017

Contato com autor responsável: Edlamar Kátia Adamy

Endereço postal: Rua Sete de Setembro 91D Sala 2, Centro, CEP 89.801-140

Chapecó-SC

E-mail: edlama.adamy@udesc.br 